\section{Cooling, Heating, Generating Power, and Recovering Waste Heat with Thermoelectric Systems}

\author{
Lon E. Bell
}

Thermoelectric materials are solid-state energy converters whose combination of thermal, electrical, and semiconducting properties allows them to be used to convert waste heat into electricity or electrical power directly into cooling and heating. These materials can be competitive with fluid-based systems, such as two-phase air-conditioning compressors or heat pumps, or used in smaller-scale applications such as in automobile seats, night-vision systems, and electrical-enclosure cooling. More widespread use of thermoelectrics requires not only improving the intrinsic energy-conversion efficiency of the materials but also implementing recent advancements in system architecture. These principles are illustrated with several proven and potential applications of thermoelectrics.

$\mathrm{B}$ road societal needs have focused attention on technologies that can reduce ozone depletion, greenhouse gas emissions, and fossil fuel usage. Thermoelectric (TE) devices, which are semiconductor systems that can directly convert electricity into thermal energy for cooling or heating or recover waste heat and convert it into electrical power, are increasingly being seen as having the potential to make important contributions to reducing $\mathrm{CO}_{2}$ and greenhouse gas emissions and providing cleaner forms of energy.

In this Review, I compare solid-state TE systems with more familiar mechanical providers of heating, cooling, and electrical power generation, such as air conditioners, refrigerators, heat pumps, heat exchangers, and turbine engines. Both classes of devices are similar in that they employ a working fluid - in TEs, this is electrical current, whereas in conventional closed-system heat engines steam or freon substitutes are the common working fluids. These classes have complementary regimes in which they can provide good performance.

Solid-state energy conversion has great appeal in terms of its simplicity as compared with systems that must compress and expand a twophase (gas/liquid) working fluid. However, except in a limited number of cases, the operational efficiencies of TE systems have fallen short of the targets needed for them to be used broadly. Nevertheless, several commercial uses have been realized, including thermal cycles for DNA synthesizers, car seat cooler/heaters, laser diode coolers, and certain low-wattage power generators. Successful applications have capitalized on the small size of these devices, their robustness in demanding environments, or their rapid response time.

Two important pathways will lead to additional applications for TEs. One will be improving the

BSST, Irwindale, CA 91706, USA. intrinsic efficiencies of TE materials, and many efforts are underway to accomplish this. Another is to improve the way in which existing TEs are currently used, which will be the main focus of this overview.

\section{Thermoelectrics As Heat Engines}

TE devices are solid-state heat engines. Unlike today's air conditioners, which use two-phase fluids such as the standard refrigerant R-134A, TE devices use electrons as their working fluid. Figure 1 demonstrates the principal effects that govern their performance.

In 1834, Peltier observed that if a current is applied across a junction of dissimilar electrically conductive materials, either heating or cooling can occur at the junction. When the current is reversed, the opposite effect is observed. Figure 1A illustrates why this occurs. Electric current is propagated by electrons in n-type materials and by holes (traveling in the opposite direction) in p-type materials, be they semiconductors, metals, or semimetals. If voltage is applied in the right direction across a $\mathrm{p}-\mathrm{n}$ junction, electron/hole pairs are created in the vicinity of the junction. Electrons will flow away from the junction in the n-type material, and holes will flow away in the p-type material. The energy to form them comes from the junction region, cooling it. On the opposite end, electrons and holes stream toward junctions where pairs recombine. This process releases energy and heats the junctions. At the bottom of Fig. 1 is a typical TE module, configured so that all junctions on one side heat and those on the other side cool.

In 1821, Seebeck noticed that the needle of a magnet is deflected in the presence of dissimilar metals that are connected (electrically in series and thermally in parallel) and exposed to a temperature gradient. The effect he observed is the basis for TE power generation. As shown in Fig. $1 \mathrm{~B}$, if the junctions at the top are heated and those at the bottom are cooled (producing a temperature differential), electron/hole pairs will be created at the hot end and absorb heat in the process. The pairs recombine and reject heat at the cold ends. A voltage potential, the Seebeck voltage, which drives the hole/electron flow, is created by the temperature difference between the hot and cold ends of the TE elements. The net voltage appears across the bottom of the TE element legs. The Seebeck effect forms the basis of the operation of TE couples (thermocouples) used extensively in temperature-measurement systems. Electrical connections can be made from the TEs to an external load to extract power.

In order for this process to be efficient, it is necessary to find materials that are good electric conductors, otherwise electron scattering generates heat on both sides of the barrier and throughout the materials. Also, the materials must be poor thermal conductors, otherwise the temperature difference that must be maintained between the hot and cold sides will produce large heat backflow. Similarly, the Seebeck effect should be maximized. Optimization of these three parameters is compromised because all three are affected by the electronic properties of the materials. Because the working fluid (electrons) conducts unwanted heat as well as electric current, and the Seebeck effect decreases as the electrical conductivity increases, it is necessary to optimize these properties simultaneously (1). The highest performance is achieved with heavily doped semiconductors, such as bismuth telluride or silicon germanium. Finally, for semiconductors, it is desirable to have a base material that can be both p- and n-type-doped, so that the same material system can be used on both sides of the junctions.

It is useful to compare the electrical current as a working fluid with the gas/liquid two-phase fluids in conventional air conditioners. The key difference that allows a refrigeration system in a building to achieve up to $60 \%$ of the maximum theoretical efficiency (as compared with $12 \%$ for TEs to date) is that cooling and heat-rejection components can be physically well separated, and large temperature differences do not lead to the high heat backflow that penalizes efficiency in TE systems.

\section{Practical Thermoelectric Devices}

In a working TE device, segments of p-type- and n-type-doped semiconductor materials, such as suitably doped bismuth telluride, are connected by shunts to form an electric circuit. The shunts are made of an excellent electrical conductor, such as copper. A voltage drives a current through the circuit, passing from one segment to another through the connecting shunts. For determining efficiency, this configuration is equivalent to the electrons passing directly from one TE material to the other. Conventional TE cooling/heating modules are constructed of pairs of TE segments, repeated about 100 times, and organized into arrays like the one shown in Fig. 1. When current 
flows within the module, one side is cooled and the other heated. If the current is reversed, the hot and cold sides reverse also. The geometry for power generators (Fig. 1B) is conceptually the same. In this case, the top side is connected to a heat source and the bottom to a heat sink. TE power generators often are similar in physical form to cooling modules except that fewer taller and thicker elements are used.

A figure of merit, $Z T$, expresses the efficiency of the p-type and n-type materials that make up a TE couple. The parameter $Z$ is the square of the Seebeck voltage per unit of temperature, multiplied by the electrical conductivity and divided by the thermal conductivity, and $T$ is the absolute temperature. In today's best commercial TE cooling/heating modules, $Z T$ is about 1.0, and in air-conditioning applications is about one-quarter as efficient as a typical conventional system, such as one that uses R-134A. Ideal TE system efficiency increases nonlinearly with $Z T$, so that to double efficiency, $Z T$ has to increase to about 2.2. To achieve a fourfold increase (to equal the efficiency exhibited by today's two-phase refrigerants), $Z T$ would need to increase more substantially to about 9.2.
To maximize power-generation efficiency, $Z T$ should be as high as possible, and the temperature differential between the hot and cold sides should be as large as possible. The material properties that make up $Z$ vary with temperature, so that materials exhibit optimum performance over a relatively narrow temperature range. As a result, in order to maximize the efficiency of powergeneration modules, individual TE elements are usually formed from two and sometimes three different TE materials laminated together in the direction of current flow to form segmented elements. Each TE material in the laminate structure is chosen to have superior performance over the range of its temperature exposure (2). For effective waste heat recovery from vehicle exhaust (an operating condition with about a $350^{\circ} \mathrm{C}$ temperature differential), the efficiency needs to be about $10 \%$ and the corresponding average $Z T$ should be about 1.25 to increase mileage up to $10 \%$. For primary power generation, the net efficiency needs to be about $20 \%$ and have an average $Z T$ of 1.5 or greater (at an $800^{\circ} \mathrm{C}$ temperature differential).

Given that the merits of solid-state energy conversion are easily understood and accepted, why are TE devices not more broadly used? The

A Cooling/heating
(Peltier effect)

B Power generation (Seebeck effect)
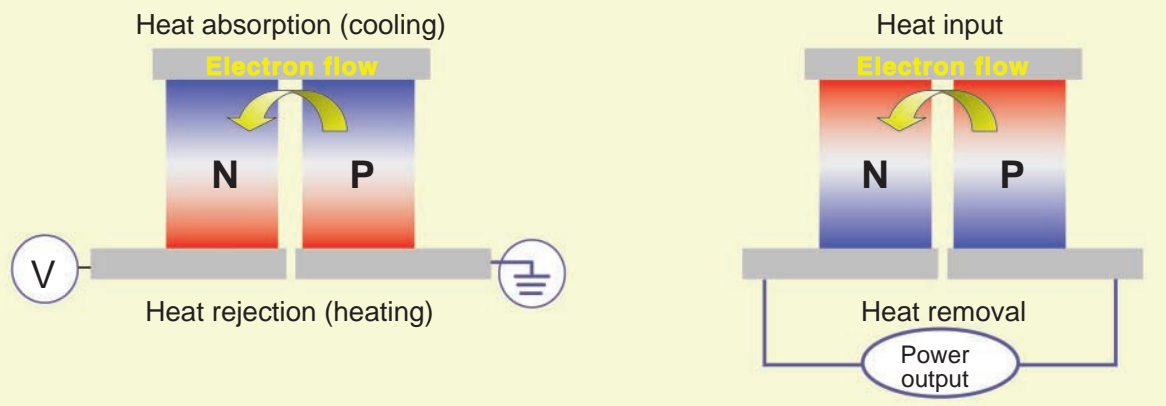

C

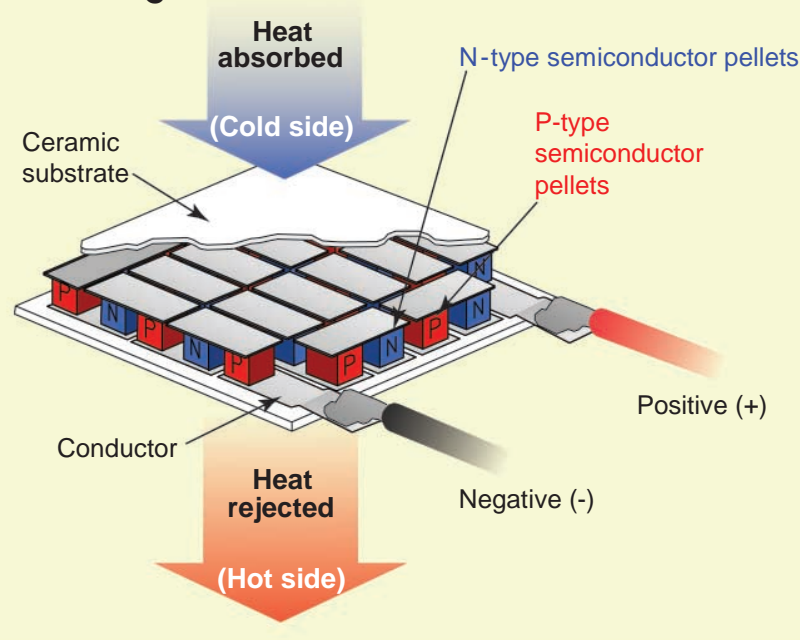

Fig. 1. TE heat engines. (A) When current is run across a TE junction, it heats or cools through the Peltier effect, depending on the direction of the current flow. (B) When heat flows across the junction, electrical current is generated through the Seebeck effect. (C) Practical TE generators connect large numbers of junctions in series to increase operating voltage and spread heat flow. principal reason is that efficiency has been too low to be economically competitive. Use in largescale air conditioners for homes and commercial buildings falls into this category. Besides low efficiency, a second reason is that the cost of traditional TE modules per watt of cooling, heating, or power generation has been too high to allow the displacement of existing technologies, with the exception of those few applications in which the beneficial characteristic of being solid state outweighs cost and performance limitations. A lack of design knowledge and design tools has created yet another barrier to broader usage. In part, this is to be expected because the technology has a legacy of low efficiency and high cost, and the small number of applications has limited the development of commercially designed software.

\section{Improving Performance}

In 1993, the U.S. government's Office of Naval Research and Defense Advanced Research Projects Agency asked interested researchers to propose pathways to improve $Z T$ for cooling and heating applications (3). A specific interest was to determine whether the then-emerging nanotechnology and its potential quantum-scale synthesis could lead to new superior TE materials. In 1993, Hicks and Dresselhouse published a theoretical model predicting the effect on $Z T$ of confining electrons to two-dimensional quantum wells (4). They calculated that the Seebeck coefficient could be increased and the thermal conductivity could be suppressed. The promise of this concept and other ideas from within the TE community led the U.S. government to fund several innovative approaches in the mid-1990s. This initiative set in motion a substantial increase in both theoretical and TE-material developmental research. By 2001, Venkatasubramanian of Research Triangle Institute announced achievement of a room-temperature $Z T$ of about 2.4 for a nanoscale structure made by alternating layers of two TE materials that both enhanced the Seebeck coefficient and suppressed thermal conductivity (5). The next year, Harman of Lincoln Laboratory published results claiming a $Z T$ of up to 3.2 at about $300^{\circ} \mathrm{C}$ for a material with nanoscale inclusions that dramatically reduced thermal conductivity (6). In 2003, Kanatzidis at Michigan State University led a team in the development of a complex bulk tertiary material with a $Z T$ of at least 1.4 at $500^{\circ} \mathrm{C}$ (7). Recently, Heremans at Ohio State University and an international team claimed reaching a ZT of 1.5 at $500^{\circ} \mathrm{C}(8)$. Despite these promising results, efficiency gains at the device level have yet to be demonstrated. The scaling of the nanomaterials has proven to be quite difficult and is still in the development stage. The bulk material has yet to be made commercially available.

The theoretical and nanoscale experimental results led to increased interest in pursuing applications enabled by the promised material advancements. At the same time as the material gains were forthcoming, attempts were made to identify other opportunities to achieve system-level performance gains. In total, four sources for these opportunities 
can be identified. The first is an obvious one: Increase the material ZT. The second is to look to continuous improvement in design optimization and to reduce parasitic losses by using nontraditional materials in device fabrication. These opportunities lead to efficiency gains of up to $25 \%$; the potential for large gains is limited because most early uses of TE devices were for aerospace applications and great efforts had already been devoted to design optimization.
The system is suited for use with a working fluid, such as air and water. Flow patterns are similar to those of counterflow heat exchangers. However, in this case, the heat transported from one fluid to the other is modified by the TE engines as it passes through the system. In cooling/heating mode, the TE elements boost the heat quality so that one of the opposing fluid streams is heated and the other is cooled. For the conditions il-
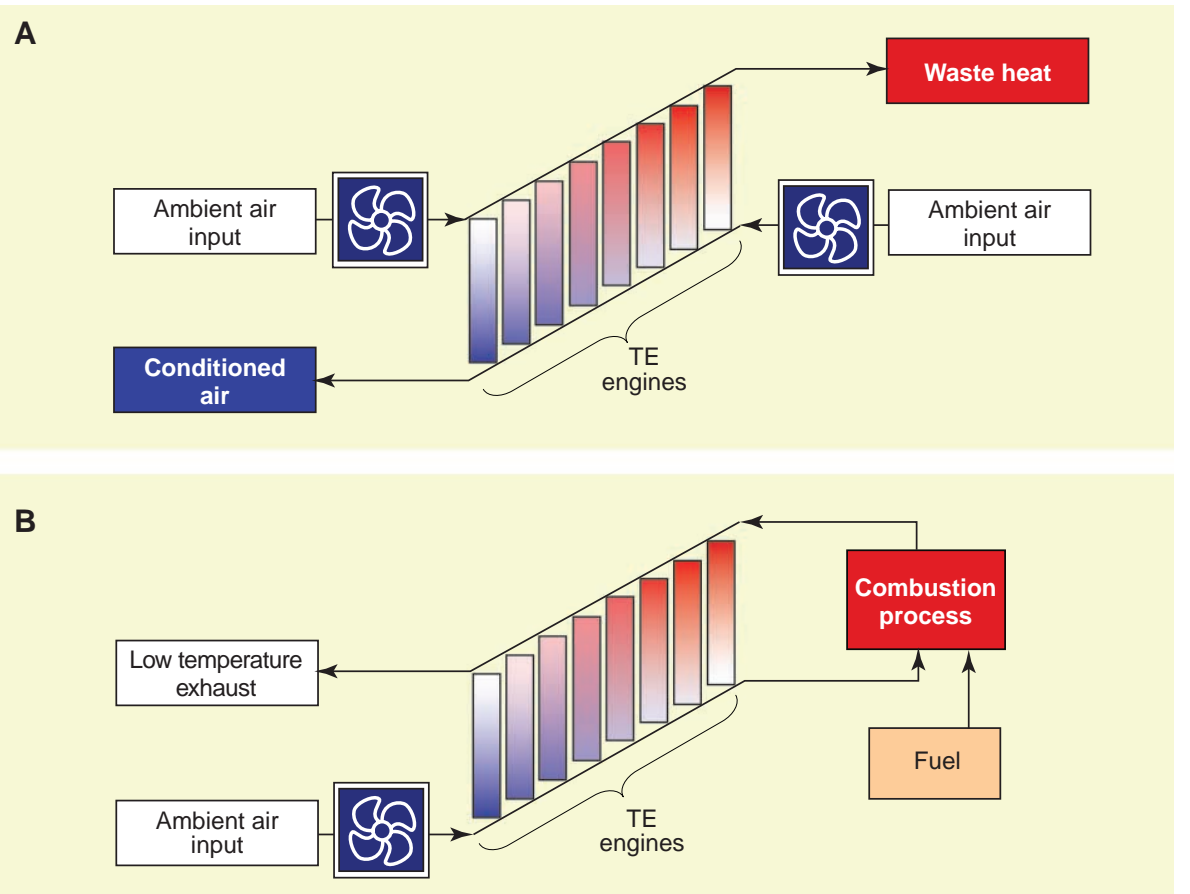

Fig. 2. Thermodynamic cycles. By optimizing each element along the thermal gradient, the engine resembles a gas turbine engine (the high-efficiency Brayton cycle) rather than the less efficient diesel cycle, in which the temperature and pressure conditions of every element (TE junction or combustion cylinder) are the same. This approach is shown for heating and cooling in (A) and for power generation in (B).

The third is to determine whether alternative thermodynamic cycles could be used to improve efficiency. In thermodynamic terms, each $\mathrm{p}$ and $\mathrm{n}$ TE couple (Fig. 1) is a separate heat engine and, in principle, could operate independently of the other engines that make up a TE device. If each engine could operate optimally (that is, at the ideal temperature and current), system-level efficiency could increase. The analog is to compare the efficiency of two common heat engines that burn oil. In a diesel engine, each cylinder is an independent heat engine, but all cylinders operate at the same temperature and pressure conditions, the diesel cycle. The engine delivers about 30 to $45 \%$ efficiency. In contrast, in a turbine engine, such as is used in municipal electric power-generation systems, every stage of the compressor and expansion sections operates optimally for the working-fluid conditions at each point. This is a regenerative Brayton cycle, and efficiency in modern systems is about 60 to $65 \%$, nearly double that of the diesel cycle. We developed a cycle analogous to the Brayton cycle, in which the TE engines are arranged as shown in Fig. 2A (9).

parasitic losses from the electrical connections (shunts) between TE elements than does the traditional configuration shown in Fig. 3A. Maximum benefit occurs if the stack design is used in combination with a reduction in the electrical resistance at the TE-material/shunt interfaces.

Other parasitic losses tend to increase when less TE material is used, so TE material volume can be lowered to the point where the benefits of the reduced resistance inherent in the stack geometry equal the increased losses from other sources. Under many practical operating conditions, the weight of TE material used can be reduced by a factor of 6 to a factor of 25 (12). By employing the design technology behind this configuration, TE material costs for high-capacity systems are generally much lower. For example, TE cooling and heating systems with the traditional configuration are cost-competitive up to about 400 thermal watts of output, but increases to about $4000 \mathrm{~W}$ with the stack design. Power generators have been restricted to uses in harsh, remote environments where reliability justifies higher costs. With substantial reductions in TE material usage, a broader spectrum of commercial applications becomes economically viable.

\section{Present Applications}

TE-powered devices have been in production since the bismuth telluride-based room-temperature materials were developed in the late 1950s. As with many innovative technologies that offer new functionality (in this case, refrigerators and heat engines the size of matchboxes), military applications developed first. TEs are used to produce $-80^{\circ} \mathrm{C}$ temperatures to operate the sensors in infrared imaging systems for heat-seeking missiles and night-vision systems. The development of silicon germanium high-temperature power-generation materials led directly to the production of heat engines for space applications with no moving parts that could operate in the absence of sunlight. Solar cells, another type of heat engine, are effective and can be used as far as the orbit of Mars, but beyond that distance the solar radiant flux is not adequate to power spacecraft. All power sources for U.S. and former-USSR deep-space probes have used TE heat engines to convert heat generated by nuclear fissile material to electricity (13).

More-recent applications take advantage of lowered costs and greater yields made possible by adopting semiconductor manufacturing processes for fabricating TE materials and devices. TE-powered devices are now in mass production for cooling, heating, and temperature-control applications in several important markets. Miniature TE modules keep laser diodes at constant temperature to stabilize operating wavelengths (14). Biological assaying has been revolutionized by the development of polymerase chain reaction (PCR) systems, and PCR systems use TE devices to thermally cycle microliter quantities of enzymatic reactions through exact series of temperature cycles (15). The process is used to multiply specific sequences of DNA material for analytical testing purposes. 
Climate-control seat (CCS) systems have been developed to provide rapid seat cooling in the summer and equally fast heating in the winter (16). The CCS is being installed in more than 500,000 vehicles a year. It increases passenger comfort and is beginning to be used to provide a degree of comfort when the vehicle engine is off. Fuel consumption is reduced in hybrid vehicles in hot driving conditions because the engine can be turned off when the vehicle is coasting, braking, or stopped.

Portable beverage and picnic coolers were an early commercial application that combined the small size, light weight, and electric operation that characterize TEs to open a new market for ice-free portable cooling systems (17). Product offerings have since expanded to include coolers that are quiet and vibration-free. They have proven popular for replacing traditional vapor-compressor refrigeration in wine-storage cabinets, hotel room mini-refrigerators, and office water coolers.

circuits allow and hence create a hazard, which now can be averted while giving the user added heating power and the benefits of cooling.

Microprocessor electronic TE-based cooling systems have seen limited application as add-ons to boost personal-computer clock speeds. Present TE systems do not demonstrate sufficient performance gains for acceptance by the general personal-computer market. However, TE cooling of small electronic enclosures, such as those used to cool the various low-power computer boards that control industrial equipment, is efficient and cost-effective. The systems have a long history of successful (if limited) application where reliability is critical and cooling capacity can be limited to less than $1000 \mathrm{~W}(19)$.

Present TE power-generation systems have been limited to uses for which their durability and maintenance-free operation dominate other performance criteria. Examples of important uses are power for remote data communication systems for

A

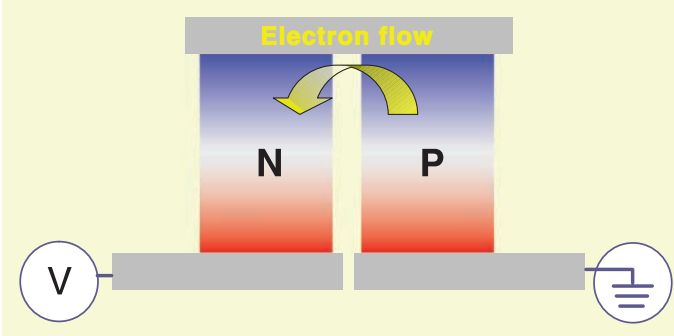

Current and heat flow

in the same general vertical direction

Long current path

through shunts

Tall, narrow TE elements
B

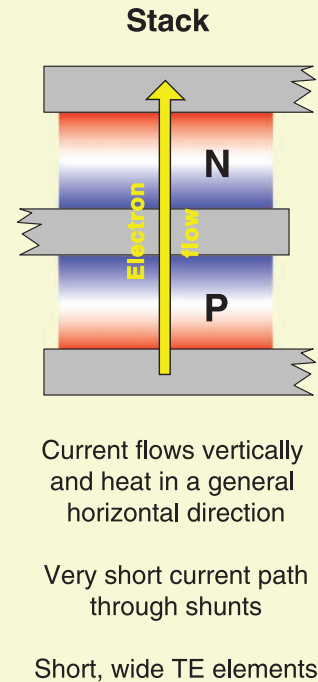

Fig. 3. Alternative TE junction geometries. (A) A traditional junction. Current and heat flow in the same general direction, and there is a long current path through shunts and tall narrow TE elements. (B) A stack junction. The current flow is perpendicular to the heat flow, the current path is minimized through shunts, and the TE elements are short and wide.

Personal temperature-control systems that provide cooling as well as heating for the office environment have also come onto the market. A desktop unit uses the high-efficiency thermodynamic cycle to condition a person's immediate environment while keeping input electrical power to that of a personal computer and within industry limits for power consumption by office furnishings and electrical outlets (18). For this and many other applications, the TE systems, when used in the heating mode, operate as heat pumps and not simply as resistive heaters. Efficiency is about two and a half times that of the traditional office resistance heaters. Usually, traditional heaters draw more electrical power than the ratings for cubicle oil and gas pipelines, polar weather station power generators, and cathodic protection for oil drilling platforms (20). TE generators are chosen for these applications because of their proven reliability (often maintenance-free operation for 20 years), durability under extreme conditions, and very little if any performance degradation over their operating life.

\section{Future Applications}

The separate technological advances in materials, cycles, and power density can be combined readily to compound benefits. More-efficient cycles are coming into use in automotive, electronic enclosure, and personal climate-control applications.
As higher-ZT materials become available, they should be able to be incorporated into existing TE designs with relatively little modification. Performance will be enhanced for all of these uses to the extent that conventional devices can be replaced with no operating-cost increase. However, the most exciting prospects for TE technology are that new uses will be enabled that have beneficial impacts on the environment.

If the average $Z T$ reaches 2, room, home, and commercial solid-state heating, ventilating, and air-cooling systems become practical. The systems would replace R-134A, which has a greenhouse gas equivalence of 1430 times that of $\mathrm{CO}_{2}$ (21), with electric current as the working fluid. Not only would the systems have zero $\mathrm{CO}_{2}$ equivalency from leakage and refrigerant disposal, but they would have exceptional heat-pumping performance, so that if the source of electrical power were green, fossil fuel usage would be eliminated in the winter as well.

Recently published review articles give a comprehensive overview of trends and accomplishments in TE material developments (22-25). Other recent publications claim gains in bulk material $Z T$ of up to $40 \%$ (26) and advancements in nanostructured silicon (a poor TE material in bulk form) to performance levels at the nanoscale on par with those of today's best commercial materials $(27,28)$. Several of the new TE materials are grown as 5- to $30-\mu \mathrm{m}$-thick films $(5,6,29,30)$. Standard semiconductor fabrication methods are used to form these materials into arrays of submillimeter couples. The resulting devices exhibit thermal response times as short as $20 \mu$ s and can cool, heat, or generate electric power. In addition to integrated circuit hot-spot cooling, these properties suggest possible new applications requiring temperature change for function, such as fast DNA analysis on a nanoliter scale, continuous environmental or hazard assaying, real-time monitoring of complex biological processes, and control and power supplies for remote-sensing systems.

Because of their ruggedness, portability, and ready ability to be electrically powered, TE systems should provide more-efficient and betterperforming temperature control in vehicles of many types, including cars, trucks, trains, and aircraft. The advantages, in addition to eliminating unfriendly refrigerants, would again be that the very efficient cooling and heating would be contained in the same package and operate with the same controls. At a $Z T$ of 2, cooling and temperature control of microprocessors, communications circuitry, electro-optical systems, and other electronic components become attractive. The clock speed and operating life of many chip circuits decreases rapidly with increased temperature, so that effective thermal management becomes beneficial on several counts (31).

Average $Z T$ in the range from 1.5 to 2 would enable substantial waste-heat harvesting and primary power-generation applications. Various government-sponsored programs are underway in the United States and Japan to increase vehicle 
mileage by converting a fraction of the heat in the exhaust systems of trucks and cars to electric power $(32,33)$. The power would be available to drive power steering, brakes, water pumps, turbochargers, and other vehicle subsystems electrically. Less electrical power would need to be produced by the alternator, which has efficiency losses caused by the load these subsystems put on engines today. On average, the electrical subsystems weigh less and can be positioned more favorably in the vehicle away from the engine, so that secondary but still important system-level gains occur. All of these factors combine to improve mileage and reduce costs. Estimates vary depending on the degree of system integration and on driving conditions, but the U.S. Department of Energy target of $10 \%$ fuel reduction appears to be within reach at these higher $Z T$ levels (34).

Gains of about 5 to $10 \%$ would be possible in diesel-powered cogenerators that are becoming widely used for onsite power generation in developed countries and for 5000 - to $20,000-\mathrm{W}$ primary generators in developing countries. In another proposed cogenerator concept, the solar spectrum is split into shorter wavelengths that yield high photovoltaic-conversion efficiency and longer wavelengths that heat a TE generator (35). Recent studies by Pacific Northwest National Laboratory suggest that industrial waste-heat recovery in aluminum smelting, glass manufacture, and cement production is practical at a $Z T$ of 2 (36). At the same $Z T$, it appears possible to replace small internal combustion engines such as those used in lawn mowers, blowers, and small outboard motorboats with external combustion TE engines. These engines would be very quiet and nearly vibration-free. They could burn a wide spectrum of fuels, such as propane, butane, liquified natural gas, and alcohols, and would not necessarily depend on fossil oil as a fuel source.

\section{Outlook}

Until recently, TE technology has languished despite the astonishing gains made in electronics, photonics, and other solid-state fields. Now, 15 years after U.S. government initiatives spurred resurgence in TE research, substantial progress is evident. More-efficient thermodynamic cycles and designs that reduce material costs are coming into commercial production. If the final enabling advancement, higher $Z T$ in TE materials, is realized, gas-emission-free solid-state home, industrial, and automotive air conditioning and heating would become practical. In power generation, fuel consumption and $\mathrm{CO}_{2}$ emissions would be reduced by electric power production from vehicle exhaust. Industrial waste-heat recovery systems could reduce emissions by providing supplemental electrical power without burning additional fossil fuel. The question is, Is TE technology on a path to overcome the historic limitations of low efficiency and high cost per watt of power conversion that have limited its applications in the past? If so, TE solid-state heat engines could well play a crucial role in addressing some of the sustainability issues we face today.

\section{References and Notes}

1. S. W. Angrist, Direct Energy Conversion (Allyn and Bacon, Boston, MA, 1965), chap. 4, pp. 144-150.

2. G. L. Snyder, Appl. Phys. Lett. 84, 2436 (2004).

3. C. B. Vining, paper presented at the European Conference on Thermoelectrics, Odessa, Ukraine, 10 to 12 September 2007.

4. L. D. Hicks, M. S. Dresselhause, Phys. Rev. B 47, 16631 (1993).

5. R. Venkatasubramanian et al., Nature 413, 597 (2001).

6. T. C. Harman et al., Science 297, 2229 (2002).

7. K. F. Hsu et al., Science 303, 818 (2004).

8. J. P. Heremans et al., Science 321, 554 (2008).

9. L. E. Bell, paper presented at the 21st International Conference on Thermoelectrics, Long Beach, CA, 25 to 29 August 2002.

10. R. W. Diller, Y. Chang, paper presented at the 21st International Conference on Thermoelectrics, Long Beach, CA, 25 to 29 August 2002.

11. L. E. Bell, paper presented at the 22nd International Conference on Thermoelectrics, Hérault, France, 17 to 21 August 2003.

12. L. E. Bell, paper presented at the 23rd International Conference on Thermoelectrics, Adelaide, Australia, 25 to 29 July 2004.

13. R. D. Abelson, Thermoelectrics Handbook (CRC Press, Boca Raton, FL, 2006), chap. 56, pp. 1-29.

14. Marlow Industries, www.marlow.com.
15. Global Medical Instrumentation; product details are at www.gmi-inc.com/BioTechLab/mjresearchptc100.htm.

16. ]. Lofy, L. E. Bell, paper presented at the 21st International Conference on Thermoelectrics, Long Beach, CA, 25 to 29 August 2002.

17. Igloo; product details are at www.igloo-store.com/ product detail.asp?T1=IGL+COOL+CHILL+40+BL\&HDR=t\& .

18. "Blowing Hot and Cold," Forbes, www.forbes.com/ leadership/2008/01/15/temperature-officeworkplace-lead-careers-cx_tw_0115bizbasics_slide_ 3.html?thisSpeed $=15000$.

19. G. S. Mikalauskis, "Selecting a Thermoelectric Cooler," www.electronicproducts.com/ShowPage. asp?Filename=teca .may2004.html.

20. Global Thermoelectric, "Thermoelectric Generators for Cathodic Protection," www.farwestcorrosion.com/fwst/ dcpower/global01.htm.

21. P. Forester et. al., in Climate Change 2007: The Physical Science Basis, Contribution of Working Group I to the Fourth Assessment Report of the Intergovernmental Panel on Climate Change (Cambridge Univ. Press, New York, 2007), p. 212.

22. F. DiSalvo, Science 285, 703 (1999).

23. B. Sales, Science 295, 1248 (2002).

24. G. J. Snyder, E. Toberer, Nat. Mater. 7, 105 (2008).

25. T. Tritt, M. A. Subramanian, MRS Bull. 31, 188 (2006).

26. B. Poudel et al., Science 320, 634 (2008).

27. A. Hochbaum et al., Nature 451, 163 (2008).

28. A. Boukai et al., Nature 451, 168 (2008).

29. H. Boettner et al., Thermoelectrics Handbook (CRC Press, Boca Raton, FL, 2006), chap. 46, pp 1-18.

30. ]. Bowers et al., Material Research Society Fall Meeting (Material Research Society, Boston, MA, 2007) 1044, p. U10-06.

31. A. Jain, S. Ramanathan, paper presented at the Intersociety Conference on Thermal and Thermomechanical Phenomena in Electronic Systems, San Diego, CA, 30 May to 2 June 2006.

32. J. Bass, N. B. Elsneer, A. Leavitt, paper presented at the 13th International Conference on Thermoelectrics, Kansas City, Missouri, 30 August to 1 September 1994.

33. K. Ikoma, M. Munekiyo, K. Furuya, T. Izumi, K. Shinohara, paper presented at the 17 th International Conference on Thermoelectrics, Nagoya, Japan, 24 to 28 May 1998.

34. L. E. Bell, paper presented at the 21st International Conference on Thermoelectrics, Long Beach, CA, 25 to 29 August 2002.

35. T. Tritt, H. Bottner, L. Chen, MRS Bull. 33, 366 (2008).

36. Industrial Technologies Program, "Engineering Scoping Study of Thermoelectric Generator Systems for Industrial Waste Heat Recovery" (U.S. Department of Energy, 2006).

37. The author is president of BSST and owns stock in the company, and BSST owns patents related to this work.

10.1126/science. 1158899 